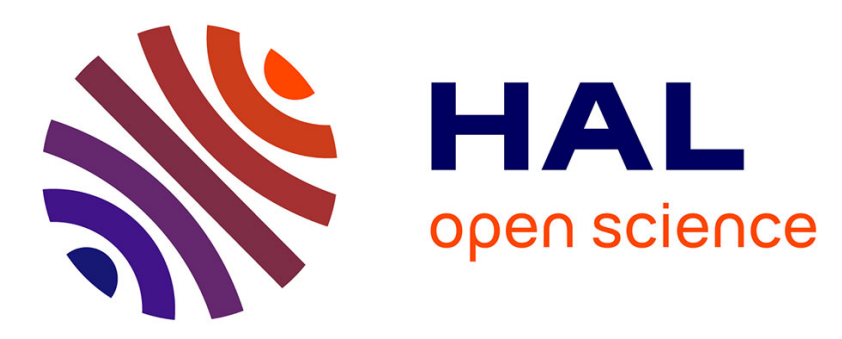

\title{
A comparison of the Hart-Smith model with the Arruda-Boyce and Gent formulations for rubber elasticity
}

Grégory Chagnon, Gilles Marckmann, Erwan Verron

\section{- To cite this version:}

Grégory Chagnon, Gilles Marckmann, Erwan Verron. A comparison of the Hart-Smith model with the Arruda-Boyce and Gent formulations for rubber elasticity. Rubber Chemistry and Technology, 2004, 77 (4), pp.724-735. 10.5254/1.3547847 . hal-01004688v2

\section{HAL Id: hal-01004688 \\ https://hal.science/hal-01004688v2}

Submitted on 3 Nov 2016

HAL is a multi-disciplinary open access archive for the deposit and dissemination of scientific research documents, whether they are published or not. The documents may come from teaching and research institutions in France or abroad, or from public or private research centers.
L'archive ouverte pluridisciplinaire HAL, est destinée au dépôt et à la diffusion de documents scientifiques de niveau recherche, publiés ou non, émanant des établissements d'enseignement et de recherche français ou étrangers, des laboratoires publics ou privés. 


\title{
A COMPARISON OF THE HART-SMITH MODEL WITH ARRUDA-BOYCE AND GENT FORMULATIONS FOR RUBBER ELASTICITY
}

\author{
G. Chagnon, G. Marckmann, E. Verron* \\ InSTITUt de ReCHERCHE EN GÉNIE CIVIL ET MÉCANIQUe \\ Ecole Centrale de Nantes, BP 92101, 44321 Nantes cedex 3, France
}

\begin{abstract}
The present paper demonstrates that the Hart-Smith constitutive model and the more recent Arruda and Boyce eight chains and Gent constitutive models are closely related. The ability of these three models to predict both small and large strain responses of rubbers is highlighted and equations that relate their material parameters are established.
\end{abstract}

\section{INTRODUCTION}

Elastomers exhibit a very complex mechanical behavior, that includes large strain, timedependent response, hysteresis, and stress-softening (Mullins effect). In the last few years, there was a significant effort to propose realistic constitutive equations for these materials. ${ }^{1-10}$ These models focus on the whole behavior of elastomers or on only one of the previously mentioned phenomena. Nevertheless, all constitutive equations are based on the general theory of hyperelasticity. ${ }^{11}$ So, consider now the non-linear elastic response of rubber-like material. It is characterized by the existence of a strain energy potential $W$. Assuming that elastomers are both isotropic and incompressible, this strain energy function only depends on the two first invariants of the left Cauchy-Green stretch tensor $\mathbf{B}$ :

$$
W=W\left(I_{1}, I_{2}\right)
$$

with:

$$
I_{1}=\operatorname{tr}(\mathbf{B}) \quad \text { and } \quad I_{2}=\left[I_{1}^{2}-\operatorname{tr}\left(\mathbf{B}^{2}\right)\right]
$$

The true stress tensor is defined by the differentiation of $W$ with respect to $\mathbf{B}$ :

$$
\sigma=-p \mathbf{I}+2 \mathbf{B} \frac{\partial W}{\partial \mathbf{B}}=-p \mathbf{I}+2\left(\frac{\partial W}{\partial I_{1}}+I_{1} \frac{\partial W}{\partial I_{2}}\right) \mathbf{B}-2 \frac{\partial W}{\partial I_{2}} \mathbf{B}^{2}
$$

This general theory being established, the major difficulty remains the choice of the function $W$. Many developments were proposed in the bibliography (see for example the review of Boyce). ${ }^{12}$

An efficient hyperelastic model can be defined by four major qualities:

- First, it should be able to accurately reproduce the whole "S" shaped response of rubbers,

- Second, the change of deformation modes should not be problematic, i.e. if the model behaves satisfactorily in uniaxial tension, it should also be quite accurate in simple shear 
or in equibiaxial extension,

- Third, the number of relevant material parameters must be as small as possible, in order to reduce the number of experimental tests needed for their identification,

- Finally, the mathematical formulation has to be quite simple to render possible the numerical implementation of the model.

Taking into account these four prerequisites, three constitutive equations can be selected: the recent models of Arruda and Boyce, ${ }^{13}$ and Gent, ${ }^{14}$ and the previous formulation of Hart-Smith. ${ }^{15}$ Through the rest of the paper, they will be referred to as AB, G and HS theories, respectively.

The aim of the present work is to establish the equations that relate these three constitutive equations. More precisely, their small and large strain characteristics will be thoroughly examined. As Boyce ${ }^{16}$ recently compared the $\mathrm{AB}$ and $\mathrm{G}$ approaches, the emphasize is laid on the equivalence of the HS model with the two other ones. In the next Section, the three strain energy functions are briefly recalled. Then, the theoretical relationships between material parameters of the models are established and the relevance of these results is demonstrated. The relevance of these results is demonstrated in Section 3. Finally, some concluding remarks are proposed.

\section{STRAIN ENERGY FUNCTIONS}

In this section, the strain energy functions that define the HS, AB and G models are briefly recalled.

\section{THE HS MODEL}

In the 60 's, Hart-Smith ${ }^{15}$ proposed an empirical form of $W$, that satisfactorily reproduces the response of rubberlike materials for the entire range of deformation endured by rubbers:

$$
W_{H S}=C_{1} \int \exp \left[C_{3}\left(I_{1}-3\right)^{2}\right] d I_{1}+C_{2} \ln \frac{I_{2}}{3}
$$

where $C_{1}, C_{2}, C_{3}$ are the three material parameters. In this equation, the first right-hand side part of $W$ describes the global response of the material. It only depends on the first strain invariant $I_{1}$. The second term that involves $I_{2}$ is based on the work of Gent and Thomas ${ }^{17}$ and improves the accuracy of the model for moderate strain (less than 150\%). It can be related to the MooneyRivlin theory that involves both $I_{1}$ and $I_{2}$ and is widely recognized for its ability to satisfactorily reproduce rubberlike behavior for small and moderate strain. ${ }^{11}$

\section{THE AB MODEL}

The model of Arruda and Boyce ${ }^{13}$ is a molecular based constitutive equation, also known as the 8 -chains model. Following Kuhn and Grün, ${ }^{18}$ the behavior of an individual chain is considered non-gaussian. In order to describe the response of the polymer network, this individual response should be integrated in an unit sphere defined by its chain density. Nevertheless, the corresponding constitutive equation necessitates numerical integration. ${ }^{19}$ So, in order to overcome this difficulty, privileged directions for chains orientation are defined by the half diagonals of a unit cube contained in the unit sphere. The corresponding strain energy function is given by: 


$$
W_{A B}=C_{R} N\left(\sqrt{\frac{I_{1}}{3 N}} \beta+\ln \frac{\beta}{\sinh \beta}\right)
$$

where $\beta=L^{-1}\left(\sqrt{I_{1} / 3 N}\right)$ with $L$ being the Langevin function defined as $L(x)=\operatorname{coth}(x)-1 / x$. In the previous equation, $C_{R}$ and $N$ are the two material parameters. Due to the non-gaussian nature of the chain modelling, $3 N$ stands for the maximum value that $I_{1}$ can reach and represents the extensibility limit of chains.

This model only depends on the first strain invariant $I_{1}$, and it is well-known that its performances at moderate strain are not very good. ${ }^{19}$ Very recently, Meissner and Matejka ${ }^{20}$ proposed the improvement of chains models by adding a function of $I_{2}$ to the strain energy. Moreover, Fried ${ }^{21}$ explained the dependance of $W$ on $I_{2}$ invoking assumptions on the network deformation.

\section{THE G MODEL}

Recently, Gent ${ }^{14}$ proposed an empirical relation for the strain energy function:

$$
W_{G}=-\frac{E}{6} \ln \left(1-\frac{I_{1}-3}{J_{m}}\right)
$$

where $E$ and $J_{m}$ are the two material parameters. $J_{m}$ represents the maximum value of $I_{1}-3$ that can be undergone by the material. It is the empirical counterpart of the extensibility limit of chains previously defined for the AB model.

\section{COMPARISON OF MATERIAL PARAMETERS}

The present section is devoted to the comparison of the three models. As these constitutive equations are supposed to be qualitatively efficient for the entire range of strains, both small and large strain responses will be compared. As mentioned above, the $I_{2}$ terms in constitutive equations can be seen as corrections of the phantom network theory. ${ }^{11}$ So, we propose to only compare the part of functions $W$ that involves $I_{1}$, i.e. the phantom part of constitutive equations. Consequently, the following HS strain energy will be considered through the rest of the paper:

$$
W_{H S}=C_{1} \int \exp \left[C_{3}\left(I_{1}-3\right)^{2}\right] d I_{1}
$$

in which only two material parameters are considered, i.e. $C_{1}$ and $C_{3}$.

Moreover, recalling that a previous work demonstrated the equivalence of the $A B$ and the $G$ approaches,${ }^{16}$ the present work emphasizes the comparison of the HS model with them.

\section{POLYNOMIAL EXPANSION}

Before explicitly comparing the models, their polynomial expansion in terms of $I_{1}$ are established. This will highly simplify the comparison of their small strain responses.

The HS strain energy function (7) can be developed into an infinite series of powers of $I_{1}$ : 


$$
W_{H S}=C_{1} \sum_{i=0}^{\infty} \frac{C_{3}^{i}}{(2 i+1) i !}\left(I_{1}-3\right)^{2 i+1}
$$

It is to note that this expression is a particular case of the general expansion proposed by Rivlin ${ }^{16}$ :

$$
W=\sum_{(r, s)=(0,0)}^{\infty} C_{r s}\left(I_{1}-3\right)^{r}\left(I_{2}-3\right)^{s}
$$

where the dependance on $I_{2}$ is not considered, said $s=0$, and only odd powers of $I_{1}$ are relevant.

The first five terms of the development of $W_{A B}$, given by Equation (5), into a series of powers of $I_{1}$ are:

$$
W_{A B}=C_{R}\left[\frac{1}{2}\left(I_{1}-3\right)+\frac{1}{20 N}\left(I_{1}^{2}-9\right)+\frac{1}{1050 N^{2}}\left(I_{1}^{3}-27\right)+\frac{19}{7000 N^{3}}\left(I_{1}^{4}-81\right)+\frac{519}{673750 N^{4}}\left(I_{1}^{5}-243\right)+\ldots\right]
$$

This expansion is also a particular case of Rivlin's series (9), but, in comparison with Equation (8), it is expressed with both odd and even powers of $I_{1}$.

Similarly to previous expansions, $W_{G}$ (see Equation (6)) can be expanded into a series of powers of $I_{1}$ :

$$
W=\frac{E}{6} \sum_{n=1}^{\infty} \frac{1}{J_{m}^{n+1}}\left(I_{1}-3\right)^{n}
$$

Here, both odd and even powers of $I_{1}$ are involved.

\section{SMALL STRAIN BEHAVIOR}

The small strain stiffness of the models are respectively defined by first terms of Equations (8), (10) and (11). Then, the three strain energy functions reduce to the neo-Hookean expres$\operatorname{sion}^{11}$ :

$$
W_{N H}=\frac{1}{2} C\left(I_{1}-3\right)
$$

where $C$ is a material parameter. Moreover, the first parameters of the HS, AB and G models are proportional. They are simply related to $C$ by:

$$
C=2 C_{1}=C_{R}=\frac{E}{3}
$$

\section{LARGE STRAIN BEHAVIOR}

As mentioned above, the three models are characterized by their ability to describe the strain-hardening of the material that takes place under large strain. This strain-hardening phe- 
nomenon is mainly due to the extensibility limit of polymer chains.

According to Equation (3) and taking into account that the considered strain energy functions do not depend on $I_{2}$, i.e. $\partial W / \partial I_{2}=0$, the stress-strain relationship reduces to:

$$
\sigma=-p \mathbf{I}+2 \frac{\partial W}{\partial I_{1}} \mathbf{B}
$$

in which only the first derivative of $W$ with respect to $I_{1}$ is involved.

In the three cases, this derivative is given by:

$$
\begin{gathered}
\frac{\partial W_{H S}}{\partial I_{1}}=C_{1} \exp \left[C_{3}\left(I_{1}-3\right)^{2}\right] \\
\frac{\partial W_{A B}}{\partial I_{1}}=\frac{C_{R} N}{2 \sqrt{3 I_{1}}} L^{-1}\left(\sqrt{\frac{I_{1}}{3 N}}\right) \\
\frac{\partial W_{G}}{\partial I_{1}}=\frac{E}{6} \frac{1}{1-\left(I_{1}-3\right) / J_{m}}
\end{gathered}
$$

Examining these equations, it is obvious that the three functions exhibit an upturn for large values of $I_{1}$. Moreover, for both $\mathrm{AB}$ and $\mathrm{G}$ models, $\partial W / \partial I_{1}$ tends asymptotically to infinity as the first invariant approaches a limiting value. Vertical asymptotes correspond with values of $I_{1}$ equal to $3 N$ and $J_{m}+3$ for $\mathrm{AB}$ and $\mathrm{G}$ constitutive equations, respectively. Thus, the second material parameters of $\mathrm{AB}$ and $\mathrm{G}$ models are obviously related by:

$$
J_{m}=3(N-1)
$$

Consider now the HS model. It does not exhibit an asymptotic behavior, because it involves an exponential-like function. Nevertheless, in order to ensure that models reproduce the same behavior at large strain, it is important that they admit similar slopes in this range of deformation. Here, the HS model is only compared with the G model, the equivalence of AB and G models being already established in Equation (18). Slopes of stress-strain curves can be expressed in terms of the second derivatives of $W$ with respect to $I_{1}$ that are:

$$
\begin{gathered}
\frac{\partial^{2} W_{H S}}{\partial I_{1}^{2}}=2 C_{1} C_{3}\left(I_{1}-3\right) \exp \left[C_{3}\left(I_{1}-3\right)^{2}\right] \\
C=2 C_{1}=C_{R}=\frac{E}{3} \\
\frac{\partial^{2} W_{G}}{\partial I_{1}^{2}}=\frac{E}{6 J_{m}} \frac{1}{\left[1-\left(I_{1}-3\right) / J_{m}\right]^{2}}
\end{gathered}
$$

Thus, to ensure that the responses of HS and G models are similar, these two derivatives should be equal. Eliminating their first material parameters, i.e. $C_{1}$ and $E$, respectively, by the use of Equation (13), it yields to: 


$$
2 C_{3}\left(I_{1}-3\right) \exp \left[C_{3}\left(I_{1}-3\right)^{2}\right]=\frac{1}{J_{m}} \frac{1}{\left[1-\left(I_{1}-3\right) / J_{m}\right]^{2}}
$$

which lies the second material parameters $C_{3}$ and $J_{m}$, and depends on $I_{1}$. This equation means that the equality of slopes depends on the considered deformation level. In order to solve this equation, it can be cast under the following form:

$$
C_{3}\left(I_{1}-3\right)^{2} \exp \left[C_{3}\left(I_{1}-3\right)^{2}\right]=\frac{I_{1}-3}{2 J_{m}} \frac{1}{\left[1-\left(I_{1}-3\right) / J_{m}\right]^{2}}
$$

The solution of this equation can be expressed in terms of the Lambert $\mathrm{W}$ function, denoted here $W^{L}$ :

$$
C_{3}\left(I_{1}-3\right)^{2}=W^{L}\left(\frac{I_{1}-3}{2 J_{m}} \frac{1}{\left[1-\left(I_{1}-3\right) / J_{m}\right]^{2}}\right)
$$

where is defined to be the function satisfying (see Corless $e t a l .^{23}$ and the references herein for details):

$$
W^{L}(x) e^{W^{L}(x)}=x
$$

So, defining now the reduced first strain invariant $\alpha$ by:

$$
\alpha=\frac{I_{1}-3}{J_{m}}
$$

where $\alpha \in[0,1]$ as $J_{m}$ represents the asymptotic value of $I_{1}-3$, and considering some basic algebraic manipulations, Equation (23) can be transformed into:

$$
C_{3} J_{m}^{2}=\frac{1}{\alpha^{2}} W^{L}\left(\frac{\alpha}{2(1-\alpha)^{2}}\right) \text { for } \alpha \in[0,1]
$$

This equation relates the second material parameters $C_{3}$ and $J_{m}$ that satisfy the equality of slopes at large strain for HS and G models. As mentioned above, it depends on $\alpha$, i.e. on the strain level.

Nevertheless, as the G model increases asymptotically to infinity in the neighborhood of $I_{1}$ $=J_{m}+3$, i.e. $\alpha=1$, and the HS model is an exponential-like function in this range of $I_{1}$, Equation (26) is not defined for $\alpha=1$. In practice, the equivalence between the two constitutive equations can only be demonstrated on a given range of $\alpha$ (or of $I_{1}$ ). In this way, a mean constant value of the function in the right-hand side of Equation (26) can be adopted to relate $C_{3}$ and $J_{m}$. This permits to omit the dependence on $\alpha$ of $C_{3} J_{m}^{2}$. Let us denote $\left[\alpha_{\min }, 1\right]$ with $\alpha_{\min }>0$, the range in which 
the HS and G models are close to. Thus, in this range, the two parameters can be linked by a constant coefficient:

$$
C_{3} J_{m}^{2}=k \text { with } k=\frac{1}{1-\alpha_{\min }} \int_{\alpha_{\min }}^{1} \frac{1}{\alpha^{2}} W^{L}\left(\frac{\alpha}{2(1-\alpha)^{2}}\right) d \alpha
$$

So, the value of $k$ depends on the range in which the equivalence of the two models is recognized. Figure 1 presents the evolution of $k$ as a function of $\alpha_{\min }$. As shown by the graph, $k$ tends to infinity as $\alpha_{\min }$ tends to 0 or 1 . The practical choice of the bound $\alpha_{\min }$ and the corresponding value $k$ will be examined in the next section for two examples.

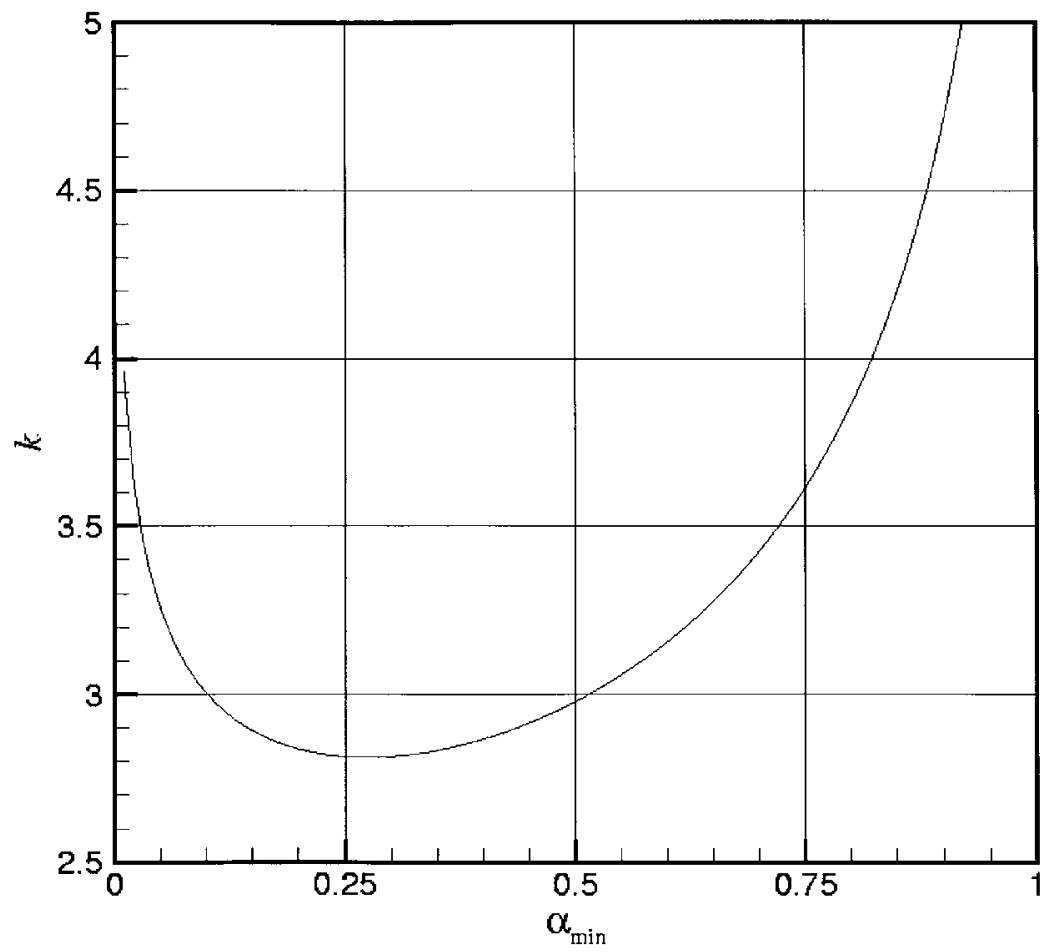

FIG. 1. - Curve of Equation (27): $k$ vs $\alpha_{\min }$.

\section{EXAMPLES}

Finally, two examples are studied to demonstrate the equivalence of the three constitutive equations, and the relevance of Equations (13), (18) and (27) that lie their material parameters.

\section{FIT OF THE G THEORY}

The first example consists in using the $\mathrm{G}$ model as a reference. In order to build fictitious materials and their corresponding data sets to be fitted, the two parameters $E$ and $J_{m}$ are considered to vary in $[0,10]$ and $[7,100]$, respectively. Values of $J_{m}$ stand for limits of extensibility between $200 \%$ and $900 \%$ in uniaxial tension.

First, $J_{m}$ is set to 60 and $E$ adopts the three values $0.1,1$ and 10. In these three cases, the function $\partial W_{G} / \partial I_{1}$ given by Equation (17) is discretized using a large number of values, and the 
corresponding stress functions of HS and AB models, respectively given by Equations (15) and (16), are fitted with these data. Fitting results are obtained using two different algorithms. First, an optimization program based on a genetic algorithm estimates approximately the material parameters. Second, these parameters are precisely determined with the help of a classical steepest descent algorithm using the genetic algorithm results as initial guess solutions. The linear relation Equation (13) between first parameters of the three models is exactly recovered for the three tests.

Second, the initial stiffness $E$ is set to 1 , and $J_{m}$ varies between 7 and 100 . First parameters of HS and $\mathrm{AB}$ models are calculated according to Equation (13), said $C_{1}=6$ and $C_{R}=3$. For the considered values of $J_{m}$, the method employed above for first parameters is used to determine both $C_{3}$ and $N$. Let us first examine the results for the $\mathrm{AB}$ model. Figure 2 shows the comparison between fitted results and the theoretical relation of Equation (18). The fitted results agree well with the theory and the linear relation between the asymptotic limit of the two models is satisfactorily recovered.

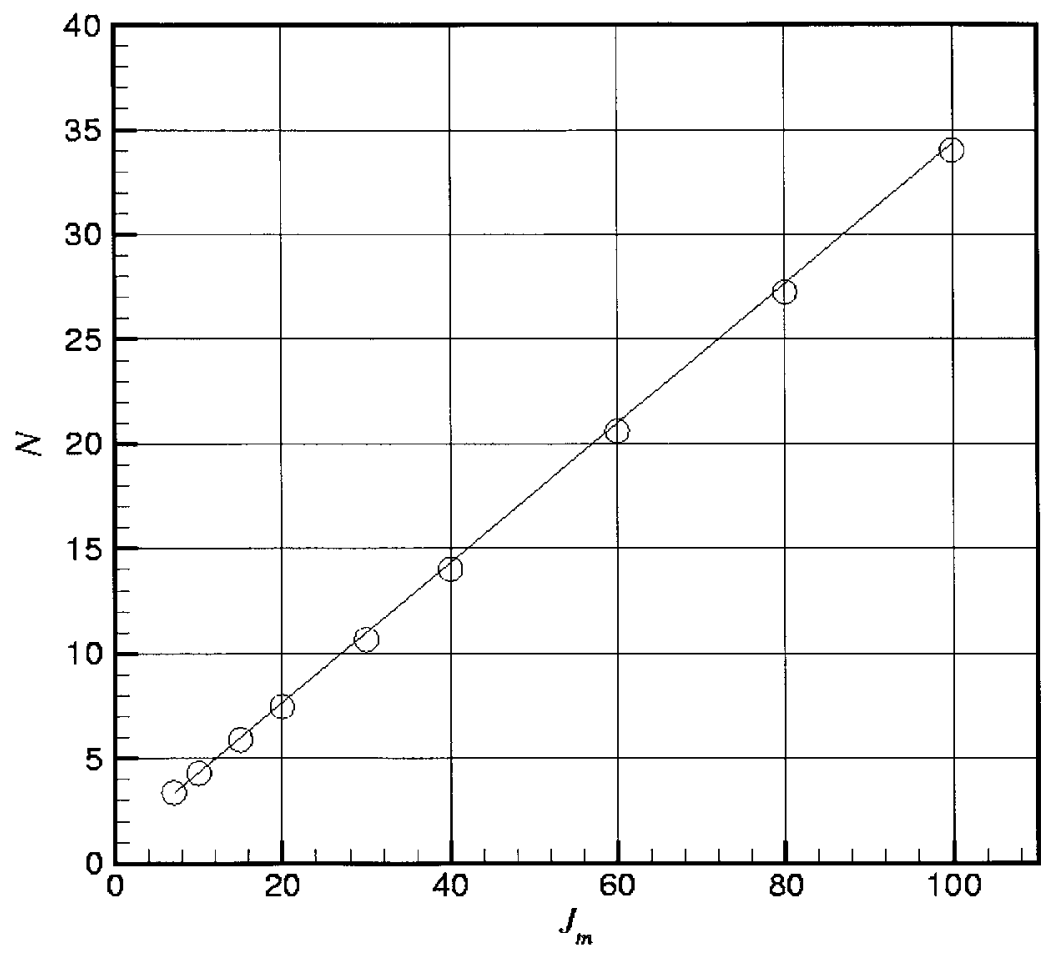

FIG. 2. - Comparison of second parameters for the $\mathrm{G}$ and $\mathrm{AB}$ models: (o) fitted results, (-) theoretical results of Equation (18).

As mentioned above, the case of the HS model is more difficult. Our goal is to verify Equation (27). Thus, the interval of $\alpha$ should be defined to calculate the integral in this equation. In order to choose the lower bound $\alpha_{\text {min }}$, we consider that slopes of HS and G models should not be compared for small strain, because only the large strain responses of these models are examined. As proposed by Kucherskii, ${ }^{24}$ the neo-Hookean formulation is valid in the first third of the deformation range. Thus, only the two thirds of the deformation range has to be retained for the large strain response, i.e. $\alpha_{\min } \geq 1 / 9$. Moreover, as models responses are highly different in the neighborhood of $\alpha=1$, because the G model tends asymptotically to infinity and the HS model grows exponentially, we consider that the equivalence should be demonstrated for more than a 
third of the deformation range, said $\alpha_{\min } \leq 4 / 9$. Consequently, according to Equation (27) and noting that $k$ does not evolve monotonously in $\alpha_{\text {min }} \in[1 / 9,4 / 9]$, the range in which $k$ varies can be easily established:

$$
2.83 \leq k \leq 2.98
$$

Finally, Figure 3 presents the comparison between fitted and theoretical results. The two boundary values of $k$ given in Equation (28) are considered. This figure shows that fitted results are satisfactorily reproduced by Equation (27), with a slight dependence on the value of $\alpha_{\text {min }}$.

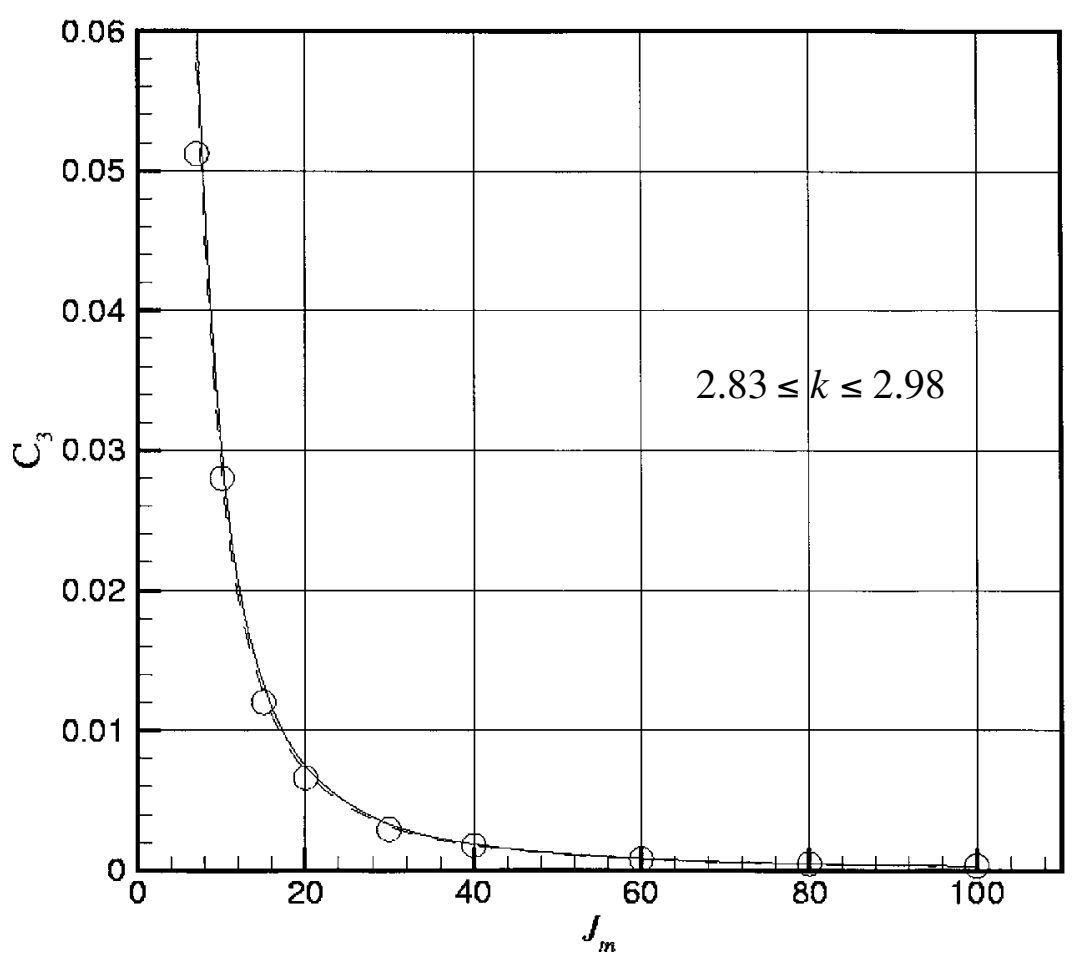

FIG. 3. - Comparison of second parameters for G and HS models: (o) fitted results, (----) theoretical results of Equation (27) for $k=2.83$, (-) theoretical results of Equation ( 27 ) for $k=2.98$.

\section{FIT OF TRELOAR'S DATA}

The second example focuses on the comparison of models for a real material. Here, experimental data of Treloar $^{25}$ are considered. The author performed a large number of experiments in different deformation modes: uniaxial tension, pure shear, equibiaxial tension (by the use of the bubble inflation technique) and biaxial tension (by combining tension and pure shear). The corresponding stress-strain responses were thoroughly examined in the past and are not recalled here. The reader can refer to Ogden ${ }^{26}$ for details.

Using these data, material parameters of the three constitutive equations are determined. The identification was performed using the four tests simultaneously. Fitted results for material parameters are presented in Table I. The corresponding results for the stress-strain responses are shown in Figure 4. In this figure, $\mathrm{X}$-axis and Y-axis of each graph correspond to relevant stretch and nominal stress for the type of experiment considered. The four graphs exhibit the similarity 
of responses for the three models and demonstrate their ability to satisfactorily reproduce different deformation modes with only two material parameters.

TABLE I

VALUes of MATERIAL PARAMETERS

\begin{tabular}{lcc}
\hline & First parameter, MPa & Second parameter \\
\hline HS model & $C_{1}=0.18$ & $C_{3}=2.710^{-4}$ \\
\hline AB model & $C_{R}=0.34$ & $N=27.9$ \\
\hline G model & $E=1.0$ & $J_{m}=92.0$ \\
\hline
\end{tabular}

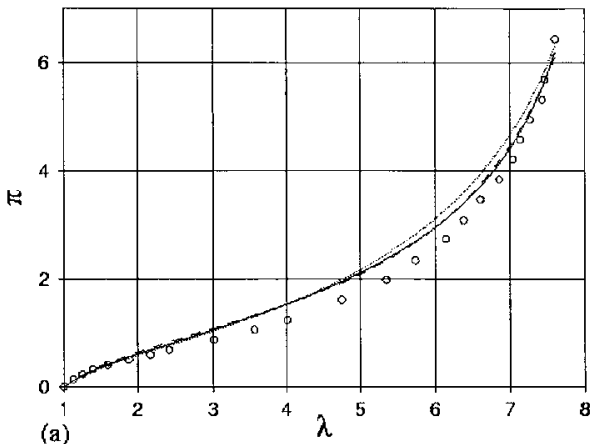

(a)

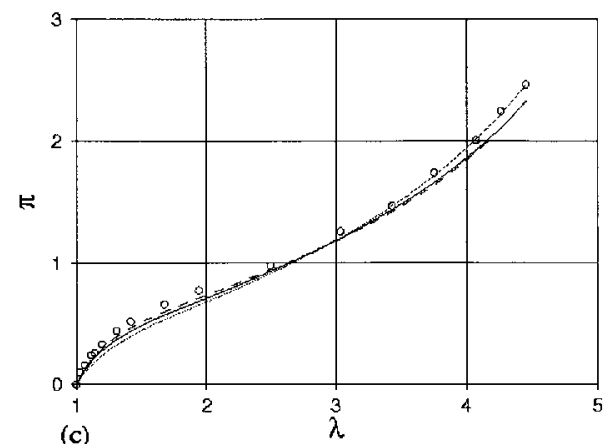

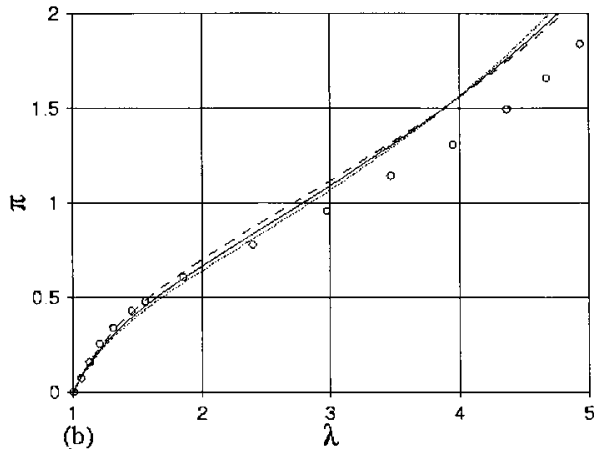

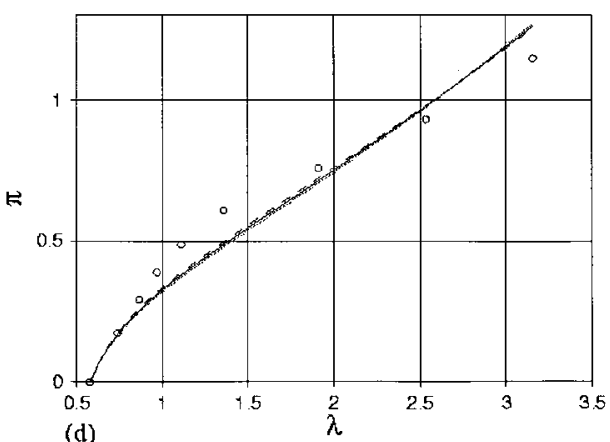

(d)

FIG. 4. - Fit of Treloar's data, (a) uniaxial tension; (b) pure shear; (c) equibiaxial tension; (d) biaxial tension; (o) experiments; (-) HS model; (----) AB model; (...) G model.

Let us now examine the relevance of the previously demonstrated relationships as applied to the parameters given in Table I. In order to simplify the discussion, G material parameters are considered as the reference and material parameters of $\mathrm{AB}$ and HS theories are compared with them.

- Concerning the first material parameter of models, we first compute $6 C_{1}=1.08$ for the HS model and $3 C_{R}=1.02$ for the $\mathrm{AB}$ theory as proposed in Equation (13). Thus, the maximum difference between these data and $E=1$ is about $8 \%$.

- Second, the large strain parameters $J_{m}$ and $N$ of $\mathrm{G}$ and $\mathrm{AB}$ models are analyzed. According to Equation (18), the theoretical value of $N$ should be $\left(J_{m}+1\right) / 3=3.10$. The difference between this predicted value and the fitted result is about $10 \%$.

- Finally, we consider the relationship between $J_{m}$ and $C_{3}$. The corresponding theoretical relation that lies these data is given in Equation (27). In order to compute $k$, the lower 
bound $\alpha_{\min }$ of the definition range should be determined. Examining experimental results for the uniaxial test shown in Figure 4(a), the small strain range, in which the neo-Hookean theory is sufficient, can be defined as $\lambda \in[1,4]$. This interval corresponds to $I_{1} \in[3,16.5]$ and the use of Equation (25) yields to $\alpha_{\min } \approx 0.14$. In regard to Equation (27), $k$ is defined by an integral from $\alpha_{\min }$ to 1 . Nevertheless, considering that the last experimental point of the uniaxial tensile test corresponds with $\lambda_{\max }=7.6$, i.e. $I_{1}=58.0$, the upper bound of the integration range is not equal to 1 but should be replaced by $\alpha_{\max }$ $=\left(I_{1 \max }-3\right) / J m \approx 0.6$. In fact, it is impossible to reach experimentally the extensibility limit of chains, because samples break for smaller stretches. Finally, the theoretical value of $k$ to be considered is given by:

$$
k=\frac{1}{0.6-0.14} \int_{0.14}^{0.6} \frac{1}{\alpha^{2}} W^{L}\left(\frac{\alpha}{2(1-\alpha)^{2}}\right) d \alpha \approx 2.69
$$

It is to note that this value is not contained in the range of Equation (28). Using this result in Equation (3), the second parameter of the HS model $C_{3}$ should have been equal to $3.18 \times 10^{-4}$. Comparing this value with the fitted result given in Table I leads to a discrepancy of $17.7 \%$. In regards with differences obtained between our theoretically predicted values and fitted results, the present developments are validated. The equivalence Equations (13), (18) and (27) can now be used to switch between $\mathrm{HS}, \mathrm{AB}$ and $\mathrm{G}$ hyperelastic constitutive equations.

\section{CONCLUSIONS}

The present work establishes the equations that relate three hyperelastic constitutive equations: the HS, AB and G models. Here, only the $I_{1}$ part of the models were compared. As proposed elsewhere, ${ }^{20}$ the accuracy of the three approaches for moderate strain can be improved by adding a function of $I_{2}$ to $W$. This can be performed with only one additional material parameter. Note that the original Hart-Smith model included this $I_{2}$ term. The HS model presents an advantage that will highly simplify the numerical implementation in finite element softwares: its large strain behavior is governed by an exponential-like function so that its tangent stiffness always exists. Moreover, the relationships established here will also simplify the identification of material parameters, because HS parameters can be now related to physical concepts through their equivalence with $\mathrm{AB}$ data.

\section{REFERENCES}

1 A. Lion, Continuum Mech. Thermodyn. 8, 153 (1996).

2 J. S. Bergstrom, M. C. Boyce, J. Mech. Phys. Solids 46, 931 (1998).

${ }^{3}$ M. Kaliske, H. Rothert, Int. J. Solids Structures 35, 2057 (1998).

${ }^{4}$ S. Reese, S. Govindjee, Int. J. Solids Structures 35, 3455 (1998).

${ }^{5}$ R. W. Ogden, D. G. Roxburgh, Proc. R. Soc. Lond. A 455, 2861 (1999).

${ }^{6}$ M. F. Beatty, S. Krishnaswamy, J. Mech. Phys. Solids 48, 1931 (2000).

${ }^{7}$ C. Miehe, J. Keck, J. Mech. Phys. Solids 48, 323 (2000).

8 A.D. Drozdov, A.I. Dorfmann, Continuum Mech. Thermodyn. 13, 183 (2001).

${ }^{9}$ G. Marckmann, E. Verron, L. Gornet, G. Chagnon, P. Charrier, P. Fort, J. Mech. Phys. Solids. 50, 2011 (2002).

${ }^{10}$ G. Chagnon, E. Verron, L. Gornet, G. Marckmann, P. Charrier, J. Mech. Phys. Solids 52, 1627 (2004).

${ }^{11}$ L.R.G. Treloar, Oxford University Press, Oxford (1975).

12 M. C. Boyce, E. M. Arruda, Rubber Chem. Technol. 73, 504 (2000). 
13 E. M. Arruda, M. C. Boyce, J. Mech. Phys. Solids 41, 389 (1993).

14 A. N. Gent, Rubber Chem. Technol. 69, 59 (1996).

${ }^{15}$ L. J. Hart-Smith, Z. Angew, Math. Phys. 17, 608 (1966).

16 M. C. Boyce, Rubber Chem. Technol. 69, 781 (1996).

17 A. N. Gent, A. G. Thomas, J. Polym. Sci. 28, 625 (1958)

18 W. Kuhn, F. Grün, Kolloideitschrift 101, 242 (1942).

${ }^{19}$ P. D. Wu, E. van der Giessen, J. Mech. Phys. Solids 41, 427 (1993).

${ }^{20}$ B. Meissner, L. Matejka, Polymer 41, 7749 (2000).

${ }^{21}$ E. Fried, J. Mech. Phys. Solids 50, 571 (2002).

${ }^{22}$ R.S. Rivlin, Phil. Trans. R. Soc. A 240, 459 (1948).

${ }^{23}$ R.M. Corless, G.H. Gonnet, D.E.G. Hare, D.J. Jeffrey, D.E. Knuth, Comput. Math. 5, 329 (1996).

${ }^{24}$ A.M. Kucherskii, Polym. Testing 22, 503 (2003).

25 L.R.G. Treloar, Trans. Faraday Soc. 40, 59 (1944).

${ }^{26}$ R.W. Ogden, Proc. R. Soc. Lond. A 326, 565 (1972). 